Authorship note: $X Z$ and $X H$ contributed equally to this work and are co-first authors.

Conflict of interest: KCN reports funding from Food Allergy Research \& Education (FARE) and End Allergies Together (EAT). KCN serves as the director of FARE and World Allergy Organization (WAO) Center of Excellence at Stanford; is an adviser at Cour Pharma; is a Cofounder of Before Brands, Alladapt, and Iggenix has obstained a research sponsorship from Nestle; is a consultant and advisory board member at Before Brands, Alladapt, Iggenix, and Probio; is a data and safety monitoring board member at NHLBl; and has

US patents for basophil testing, multifood immunotherapy and prevention, monoclonal antibody from plasmoblasts, and a device for diagnostics.

Copyright: (c) 2021, Zhou et al. This is an open access article published under the terms of the Creative Commons Attribution 4.0 International License.

Submitted: August 7, 2020

Accepted: February 10, 2021

Published: February 11, 2021

Reference information: JCI Insight. 2021;6(6):e143058.

https://doi.org/10.1172/jci.

insight.143058.

\section{Targeted DNA methylation profiling reveals epigenetic signatures in peanut allergy}

\author{
Xiaoying Zhou, Xiaorui Han, Shu-Chen Lyu, Bryan Bunning, Laurie Kost, Iris Chang, Shu Cao, \\ Vanitha Sampath, and Kari C. Nadeau
}

Sean N. Parker Center for Allergy \& Asthma Research at Stanford University and Division of Pulmonary, Allergy, and Critical Care Medicine, Stanford University, Stanford, California, USA.

\begin{abstract}
DNA methylation (DNAm) has been shown to play a role in mediating food allergy; however, the mechanism by which it does so is poorly understood. In this study, we used targeted nextgeneration bisulfite sequencing to evaluate DNAm levels in $\mathbf{1 2 5}$ targeted highly informative genomic regions containing $602 \mathrm{CpC}$ sites on 70 immune-related genes to understand whether DNAm can differentiate peanut allergy (PA) versus nonallergy (NA). We found PA-associated DNAm signatures associated with 12 genes ( 7 potentially novel to food allergy, 3 associated with Th1/Th2, and 2 associated with innate immunity), as well as DNAm signature combinations with superior diagnostic potential compared with serum peanut-specific IgE for PA versus NA. Furthermore, we found that, following peanut protein stimulation, peripheral blood mononuclear cell (PBMCs) from PA participants showed increased production of cognate cytokines compared with NA participants. The varying responses between PA and NA participants may be associated with the interaction between the modification of DNAm and the interference of environment. Using Euclidean distance analysis, we found that the distances of methylation profile comprising 12 DNAm signatures between PA and NA pairs in monozygotic (MZ) twins were smaller than those in randomly paired genetically unrelated individuals, suggesting that PA-related DNAm signatures may be associated with genetic factors.
\end{abstract}

\section{Introduction}

Food allergies affect up to $7.6 \%$ and $10.8 \%$ of children and adults in the United States, respectively $(1,2)$. Because of the life-threatening potential of anaphylaxis associated with IgE-mediated food allergies, food allergy has become a growing clinical and public health problem (1).

Food allergy is a complex immune disease influenced by an interplay of genetic variants, environmental exposures, gene-environment interactions, and epigenetic modifications (3). Epigenetic modifications have been shown as one of the mechanisms used to adapt to environmental exposures and in mediating gene-environment interactions (4). Epigenetic factors, specifically DNA methylation (DNAm), play important roles in the development of food allergy $(3,5)$. A study by Canani et al. found higher DNAm levels associated with Th1-related genes (IL10 and IFNG) and lower DNAm levels associated with Th2-related genes (IL4 and IL5) in those with cows' milk allergy compared with those tolerant to cows' milk (6). Another study found that peanut oral immunotherapy decreased DNAm of the FOXP3 gene, a gene associated with tolerogenic responses (7). The study by Martino et al. (8) showed $96 \mathrm{CpG}$ sites that predicted clinical reactivity to food challenge in food-sensitized infants. These sites overlapped with 73 protein-coding genes significantly enriched with the mitogen-activated protein (MAP) kinase canonical pathway. The same group (9) applied genome-wide DNAm to delineate epigenetic modifications in naive T cells activated by bead-bound anti-CD3/anti-CD28 in egg-allergic participants and nonatopic controls, and they found a distinct DNAm profile for genes involved in metabolic and immunological regulation of egg allergy.

These studies are encouraging, and DNAm signatures offer diagnostic and therapeutic potential for food allergy. However, DNAm signatures for food allergy differ between studies, and further research — including exploratory research to identify other potentially novel DNAm signatures - is needed. In this study, we performed targeted next-generation bisulfite sequencing (tNGBS) to evaluate DNAm levels in 125 highly informative genomic regions containing $602 \mathrm{CpG}$ sites for 70 immune-related genes. 
We performed tNGBS to evaluate DNAm levels on peripheral blood mononuclear cells (PBMCs) from 10 peanut allergy (PA) and 10 nonallergy (NA) participants, aged 5-10 years old. Among these participants, 5 pairs of participants were (PA versus NA) monozygotic (MZ) twin siblings. The other 10 participants included 5 pairs of dizygotic (DZ) twin siblings (2 pairs PA, 2 pairs NA, 1 pair of PA versus NA). The DZ twin siblings were used as randomly paired genetically unrelated individuals. MZ twins discordant for PA are rare in the population, and the inclusion of these twin samples improves the statistical power by reducing the amount of genetic and/or environmental variability. We observed that the average $\mathrm{CpG}$ methylation levels within the targeted genomic regions for 12 genes showed significant differences between PA and NA participants. PA participants also showed decreased DNAm levels at each of 5 CpG sites in the targeted serine protease inhibitor E1 (SERPINE1) region compared with NA participants. Our results indicate PA-associated DNAm signatures at targeted genomic regions are associated with 12 genes, of which 7 are potentially novel to food allergy (brain-derived neurotrophic factor [BDNF], IL17F, CXCL12, CCR7, runt-related transcription factor 1 [RUNX1], CD3e, and SERPINE1); 3 are associated with Th1/Th2 responses (IL4,IL12B, and IL2) and 2 are associated with innate immune responses (IL1B and IL6). Using Luminex assay, we found increased secretion of cytokines IL-4, IL-12B, IL-1B, IL-6, CXCL12, and BDNF and secreted protein SERPINE1 following allergen-specific stimulation in PA compared with NA participants. Using stepwise regression analysis and receiver operating characteristic (ROC) curve analysis, 3 combinations of the DNAm signatures from the initial 12 DNAm signatures were selected with top ranked Akaike information criterion (AIC) and AUC. ROC comparison analysis was performed to compare the diagnostic performance of these 3 combinations of DNAm signatures against the existing diagnostic test and serum peanut-specific IgE. Our results indicate that the combinations of DNAm signatures from the initial 12 DNAm signatures had superior diagnostic potential compared with serum peanut-specific IgE for discriminating PA versus NA. We also evaluated the similarity of 12 DNAm signatures between PA and NA participants in MZ twin sibling pairs and randomly paired genetically unrelated individuals using Euclidean distance analysis. We found smaller distances between PA and NA participants in MZ twins compared with randomly paired genetically unrelated individuals, suggesting that the 12 PA-associated DNAm signatures may be associated with genetic factors. Altogether, our results demonstrate 12 food allergy-associated DNAm signatures and differences in protein secretion in response to allergen-specific stimulation between PA and NA participants. In addition, our results indicate the diagnostic potential of DNAm signature combinations for PA and indicate genetic influences on PA-associated DNAm signatures.

\section{Results}

DNAm signatures were identified in PA. To achieve a set of food allergy-related DNAm signatures, we selected 125 highly informative genomic regions containing $602 \mathrm{CpG}$ sites for 70 immune-related genes based on a comprehensive literature review, DNAm results from our previous study (10), and the genes important immune system function as per existing immunology panel from EpigenDx Inc. (Supplemental Tables 1 and 2; supplemental material available online with this article; https://doi.org/10.1172/jci. insight.143058DS1). tNGBS was performed on PBMCs from 10 NA and 10 PA participants (Table 1). First, we compared the average $\mathrm{CpG}$ methylation levels within individual targeted genomic regions using the Wilcoxon rank sum test. Significant differences were observed in the average methylation levels in the 12 targeted genomic regions for 12 genes (IL4, IL12B, IL2, IL17F, IL1B, IL6, CXCL12, BDNF, CCR7, CD3E, RUNX1, and SERPINE1) between NA and PA participants (Figure 1, A and B) $(P<0.05$; Supplemental Table 3). Next, we compared DNAm levels at each $\mathrm{CpG}$ site in the above 12 genomic regions for 12 genes between PA and NA participants (Supplemental Table 3). Of note, the DNAm levels at each of $5 \mathrm{CpG}$ sites in the targeted genomic region (chr7:101126423-101126457) for the gene SERPINE1 were significantly decreased in PA, compared with NA participants (FDR-adjusted $P<0.1$ ) (Figure 2).

The principal component analysis (PCA) of the above 12 DNAm signatures showed that the first principal components accounted for $94.68 \%$ of the data set variation and separated PA from NA participants (Figure 1C). To further confirm that the centroid and dispersion of 2 distinct clusters were different between PA and NA samples, we carried out a permutational multivariate analysis of variance (PERMANOVA) for PCA. This test examines the contribution of variables to the separation of the data in multiple dimensional spaces, and it showed a significant $P$ value between 2 groups $(P<0.001)$.

We then compared the composition of major immune cell populations between NA and PA participants who are genetically unrelated (demographic characteristics shown in Supplemental Table 4) 
Table 1. Demographics for the peanut-allergic and nonallergic participants analyzed in the study

\begin{tabular}{|c|c|c|c|c|c|c|c|c|c|c|}
\hline Twin pair & Sample ID & $\begin{array}{c}\text { Peanut allergy } \\
\text { assessment }\end{array}$ & Zygosity & $\begin{array}{l}\text { Age at blood } \\
\text { sample } \\
\text { collection }\end{array}$ & Sex & Race & $\begin{array}{l}\text { Food } \\
\text { allergies }\end{array}$ & $\begin{array}{c}\text { Food } \\
\text { allergens }\end{array}$ & $\begin{array}{c}\text { History } \\
\text { of atopic } \\
\text { dermatitis }\end{array}$ & $\begin{array}{l}\text { History } \\
\text { of } \\
\text { asthma }\end{array}$ \\
\hline \multirow[t]{2}{*}{1} & TWN1_B & $\begin{array}{l}\text { Yes by } \\
\text { oral food } \\
\text { challenge }\end{array}$ & Monozygotic & 10 & Female & White & Allergic & $\begin{array}{c}\text { Peanut, tree } \\
\text { nuts }\end{array}$ & Yes & Yes \\
\hline & TWN1_A & No by history & Monozygotic & 10 & Female & White & Nonallergic & No & Yes & No \\
\hline 2 & TWN2_A & No by history & Monozygotic & 5 & Male & Asian & Nonallergic & No & No & No \\
\hline \multirow[t]{2}{*}{3} & TWN3_B & $\begin{array}{l}\text { Yes by } \\
\text { oral food } \\
\text { challenge }\end{array}$ & Monozygotic & 9 & Female & Black & Allergic & $\begin{array}{l}\text { Peanut, tree } \\
\text { nuts }\end{array}$ & No & Yes \\
\hline & TWN3_A & No by history & Monozygotic & 9 & Female & Black & Nonallergic & No & Yes & No \\
\hline \multirow[t]{2}{*}{5} & TWN5_B & $\begin{array}{l}\text { Yes by } \\
\text { oral food } \\
\text { challenge }\end{array}$ & Monozygotic & 5 & Male & Asian & Allergic & Peanut & No & No \\
\hline & TWN5_A & No by history & Monozygotic & 5 & Male & Asian & Nonallergic & No & No & No \\
\hline \multirow[t]{2}{*}{6} & TWN6_B & $\begin{array}{l}\text { Yes by } \\
\text { oral food } \\
\text { challenge }\end{array}$ & Dizygotic & 5 & Male & Asian & Allergic & $\begin{array}{c}\text { Peanut, tree } \\
\text { nuts }\end{array}$ & Yes & No \\
\hline & TWN6_A & No by history & Dizygotic & 5 & Female & Asian & Nonallergic & No & No & No \\
\hline \multirow[t]{2}{*}{7} & TWN7_B & No by history & Dizygotic & 6 & Male & White & Allergic & Tree nuts & No & No \\
\hline & TWN7_A & No by history & Dizygotic & 6 & Female & White & Nonallergic & No & No & No \\
\hline 8 & TWN8_A & $\begin{array}{l}\text { Yes by } \\
\text { oral food } \\
\text { challenge }\end{array}$ & Dizygotic & 4 & Female & Asian & Allergic & Peanut & No & No \\
\hline 10 & TWN10_B & $\begin{array}{l}\text { Yes by } \\
\text { oral food } \\
\text { challenge }\end{array}$ & Dizygotic & 5 & Female & White & Allergic & Peanut, egg & No & No \\
\hline
\end{tabular}

using flow cytometry and found no differences in major immune cell types, including CD3, CD4, CD8, B cells, NK cells, monocytes, and DCs between NA and PA participants (Supplemental Figure 1). Since there were no statistical differences in the cell phenotypes between groups, it is unlikely that the statistically significant differences in methylation profiles between the groups can be attributed in a major way to cell heterogeneity.

The secretion levels of the proteins encoded by the genes overlapping DNAm signatures were increased from PBMCS in $P A$ compared with NA participants after peanut stimulation. Of the above 12 genes showing significant differences in DNAm levels between PA and NA participants, there were 8 cytokine genes (IL4,IL12B, IL2, IL17F, $I L 1 B, I L 6, C X C L 12$, and $B D N F$ ) and 1 gene encoding secreted protein SERPINE1. To determine if these preexisting differential DNAm signatures were associated with expression of their cognate proteins, the PBMCs from NA and PA individuals were incubated either with or without peanut protein. After a 3-day incubation, the secretion levels of these cytokines and SERPINE1 from PBMCs in supernatants were measured using a 
A
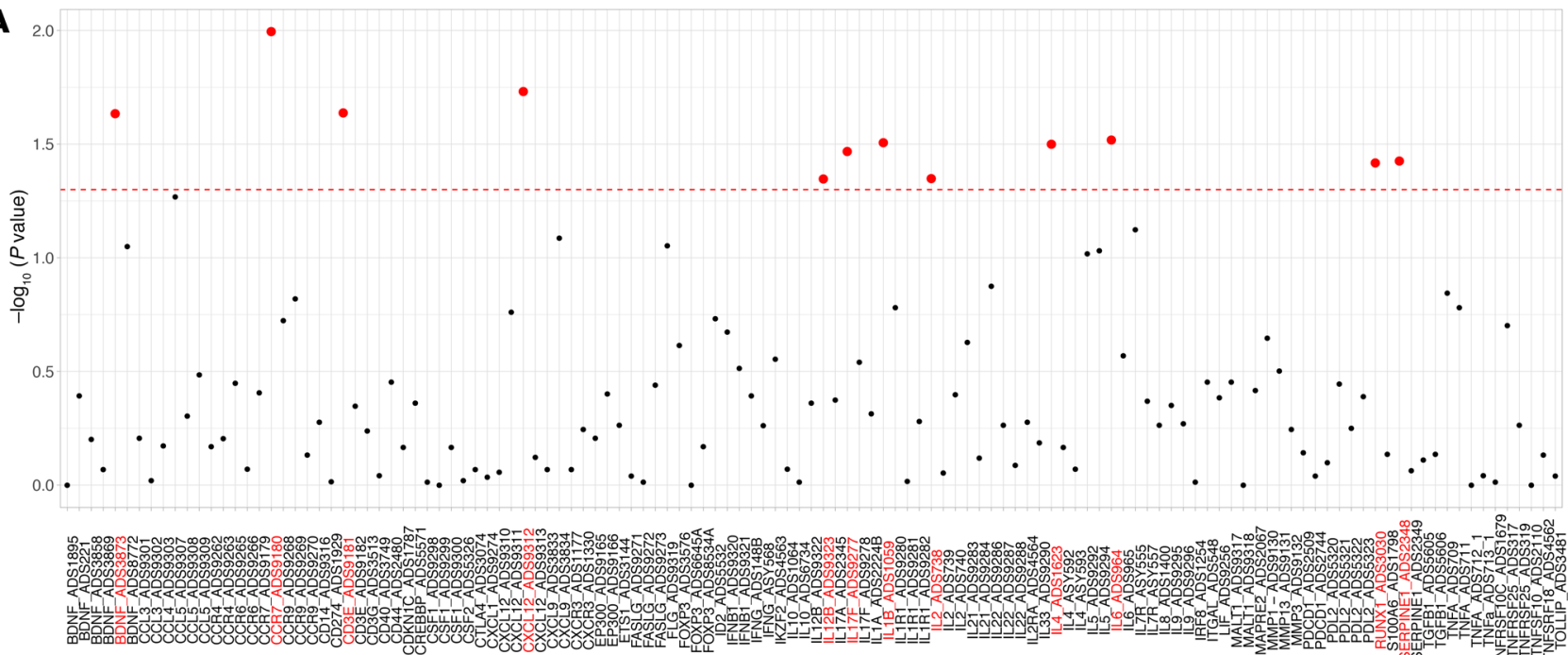

B
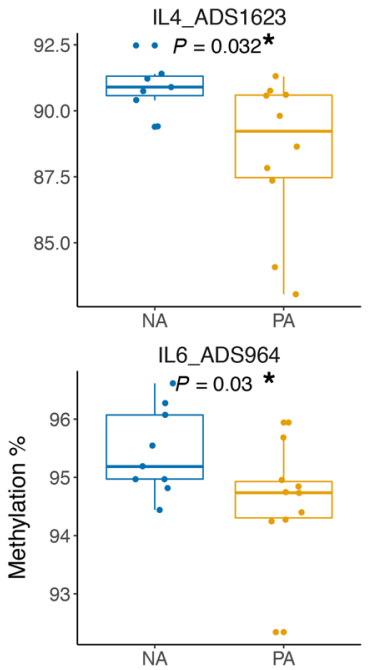

CCR7_ADS9180
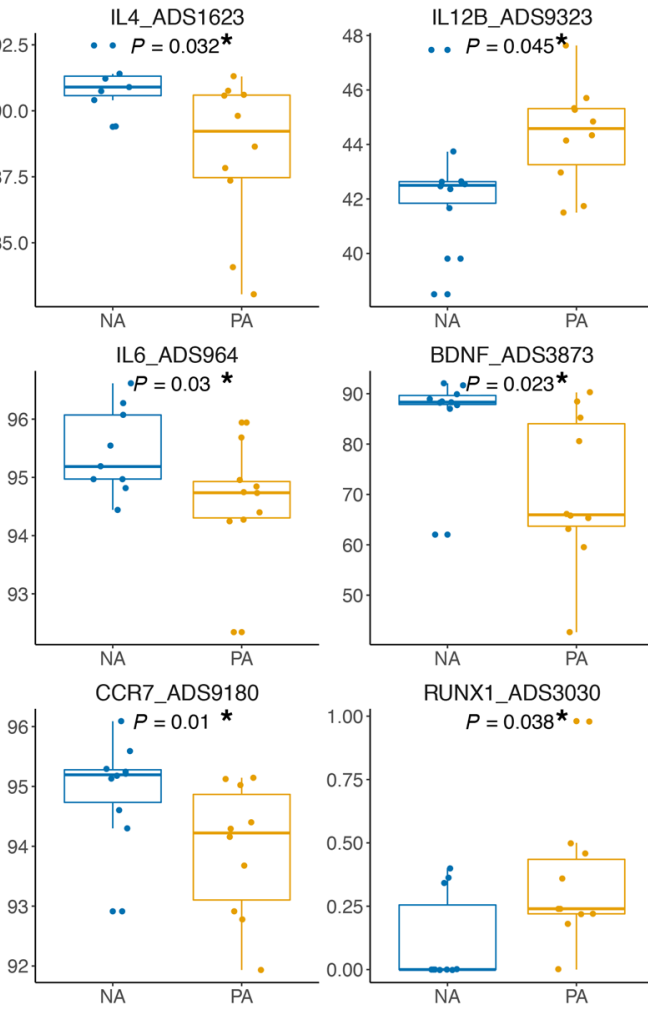

BDNF ADS3873

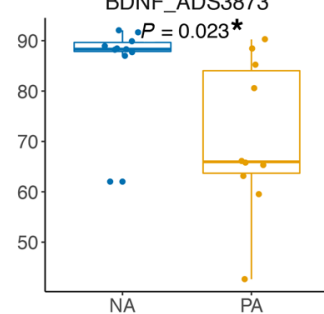

RUNX1 ADS3030
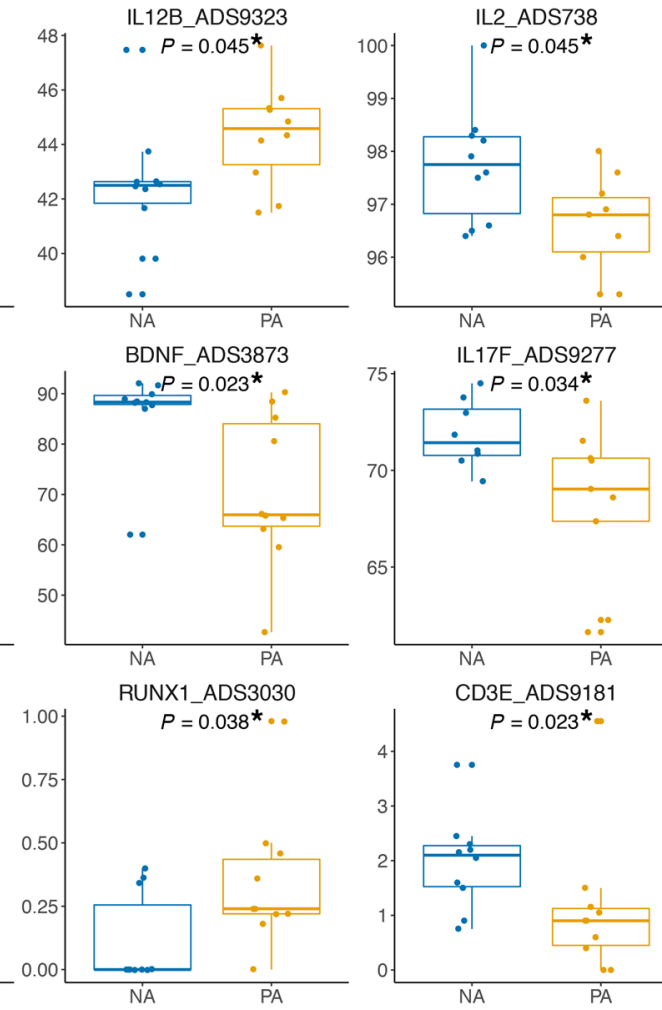

IL17F_ADS9277

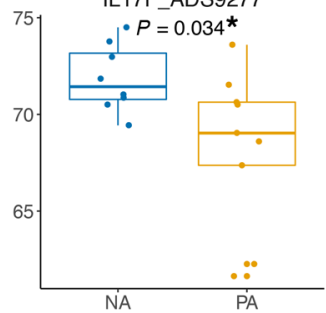

CD3E ADS9181

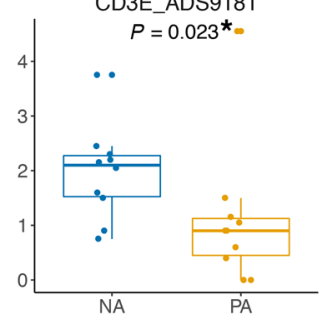

c
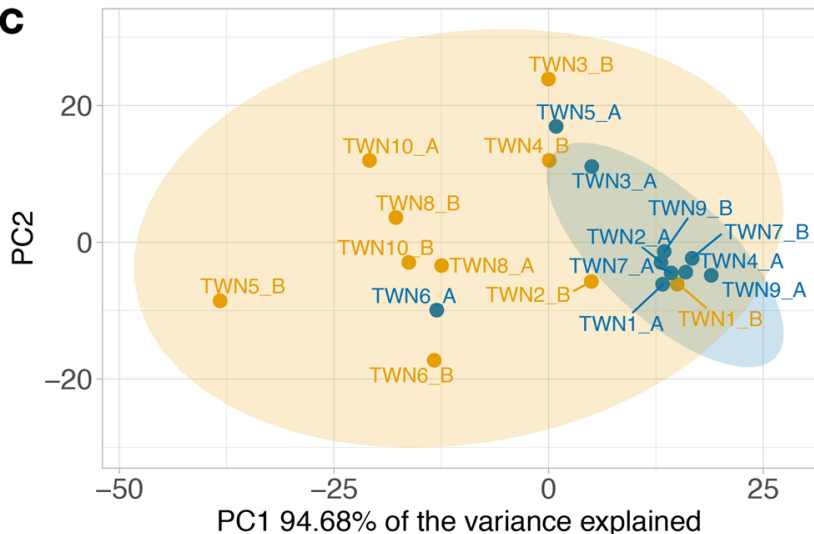

PC1 $94.68 \%$ of the variance explained

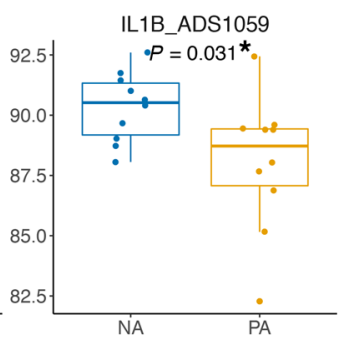

CXCL12_ADS9312

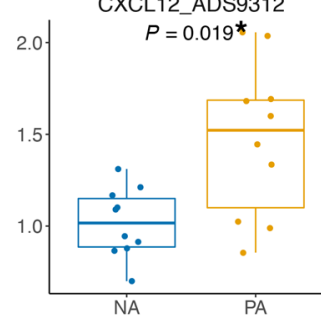

Non-Allergy (NA)

Peanut Allergy (PA)
间 Non-Allergy (NA)

Peanut Allergy (PA) 
Figure 1. The significant differences in the DNAm levels for 12 targeted genomic regions were observed between PA and NA participants. (A) The comparison analysis on the average DNAm level in each of 125 targeted genomic regions between PA $(n=10)$ and NA $(n=10)$ participants was performed using nonparametric unpaired comparison test (Wilcoxon rank sum test). The $y$ axis shows the $-\log _{10} P$ value, and the $x$ axis shows 125 targeted genomic regions. The horizontal red dashed line represents a statistical significance level of $P=0.05$. Each dot represents the $P$ value, and the red dots indicate the $P$ value less than of 0.05. (B) The box plots overlaid with dot plots show the significant differences in the average methylation levels for 12 targeted genomic regions between PA $(n=10)$ and NA $(n=10)$ participants $\left({ }^{*} P<0.05\right)$. (C) PCA of the DNAm levels in the 12 targeted genomic regions shows the 2 distinct clusters formed from NA and PA individuals. The percentage variance explained by principal component (PC) 1 is indicated. Yellow circles represent PA samples; blue circles represent NA samples.

Luminex-based assay. We found that, compared with NA participants, peanut protein-stimulated PBMCs from PA participants showed increased production of 7 cytokines (IL-4, IL-12 $\beta$, IL-2, IL-1 $\beta$, IL-6, CXCL12, and BDNF) and SERPINE1 protein (Figure 3). These results suggest that the differences in protein secretion in response to specific food allergens between PA and NA may be associated with DNAm modifications and environmental interactions.

The performance of combinations of DNAm signatures for discrimination of PA versus NA participants was superior to serum peanut-specific IgE. Next, we performed ROC analysis to calculate the AUC of 12 DNAm signatures. The AUC for 12 individual ROC curves varied from 0.77 to 0.845 for PA versus NA. Diagnostic sensitivity for individual DNAm signatures at $90 \%$ specificity ranged from $10 \%$ to $70 \%$ for PA versus NA (Figure 4A). On the basis of the assumption that each DNAm signature can be considered as a diagnostic test, we attempted to optimize DNAm signature combinations from the initial 12 DNAm signatures. We applied a stepwise (step-up) regression analysis and selected 3 models that have top-ranked AIC and AUC. The $\beta$ value (standardized regression coefficients) of the 3 models are as follow: model 1, +82.518 (CXCL12) - $2.5(B D N F)+107.287$; model 2, +134.199 $(C X C L 12)-2.230(B D N F)+12.416(C D 3 E)-10.798$; model 3, +73.62 (CXCL12) - $1.543(B D N F)-$ 1.267 (SERPINE1) + 85.039 (Table 2). To determine whether these 3 models with the combination of DNAm signatures could exceed the diagnostic potential of existing diagnostic tests, we compared the above ROC curves against peanut-specific IgE levels in serum (Figure 4B). The 3 models with the combination of the DNAm signatures had the AUC of 0.97 for model $1(C X C L 12+B D N F), 0.98$ for model $2(C X C L 12+B D N F+C D 3 E)$, and 0.98 for model $3(C X C L 12+B D N F+$ SERPINE1), respectively, compared with 0.85 for serum peanut-specific IgE (Figure $4 \mathrm{~B}$ and Table 2 ). The sensitivities at $90 \%$ specificity for discrimination of PA versus NA for 3 models with the combination of the selected DNAm signatures were 70\% (model 1), 90\% (model 2), and 90\% (model 3), respectively. These results suggest that DNAm signatures can be combined to produce highly clinically sensitive and specific DNAm panels, and the above 3 combinations of DNAm signatures have superior diagnostic potential compared with peanut-specific IgE in serum (Figure $4 \mathrm{~B}$ and Table 2).

PA-associated DNAm signatures were genetically influenced. Among the $10 \mathrm{PA}$ and $10 \mathrm{NA}$ participants, 5 pairs were PA discordant MZ twin siblings aged 5-10 years old (Table 1). Since MZ twin pairs are widely regarded as genetically identical, and young twins usually share similar environmental backgrounds, the discordant PA in MZ twin pairs are presumed to result from different epigenetic mechanisms. The epigenetic drift during the lifetime of $\mathrm{MZ}$ twin pairs has been suggested to arise from differing environmental histories $(11,12)$. In contrast, the contributions of both environmental in utero and underlying genetic factors to epigenetic profile have been suggested in neonatal epigenome for MZ twins with discordant phenotypes (13). To estimate relative contributions of the genetic factors to the epigenetic profile comprising 12 DNAm signatures, we performed a Euclidean distance-based analysis to compare the similarity of food allergy-associated epigenetic profile in the MZ twins and the randomly paired genetically unrelated individuals. The median of distances between PA and NA pairs in the MZ twins decreased by $48.28 \%$ compared with the median distances in the randomly paired genetically unrelated individuals, although the results did not reach statistical significance $(P=0.14)$ (Figure 5, A and B). The smaller distance between PA and NA participants in MZ twins suggests that PA-related DNAm signatures may be associated with genetic factors. Further studies are needed to test if this is significant. We also observed a smaller distance between MZ twins and genetically unrelated individuals in PA participants compared with NA participants; this suggests epigenetic similarities for the above 12 DNAm signatures in PA, but not NA, regardless of whether the PA participants were MZ twin siblings or genetically unrelated individuals (Figure 5, A and C). 
Transcript ID: ENST00000223095.4 CCDS: 5711 Transcript Length: 3190bp, 402aa Location: Chr7: 101,127,089-101,139,266(+)
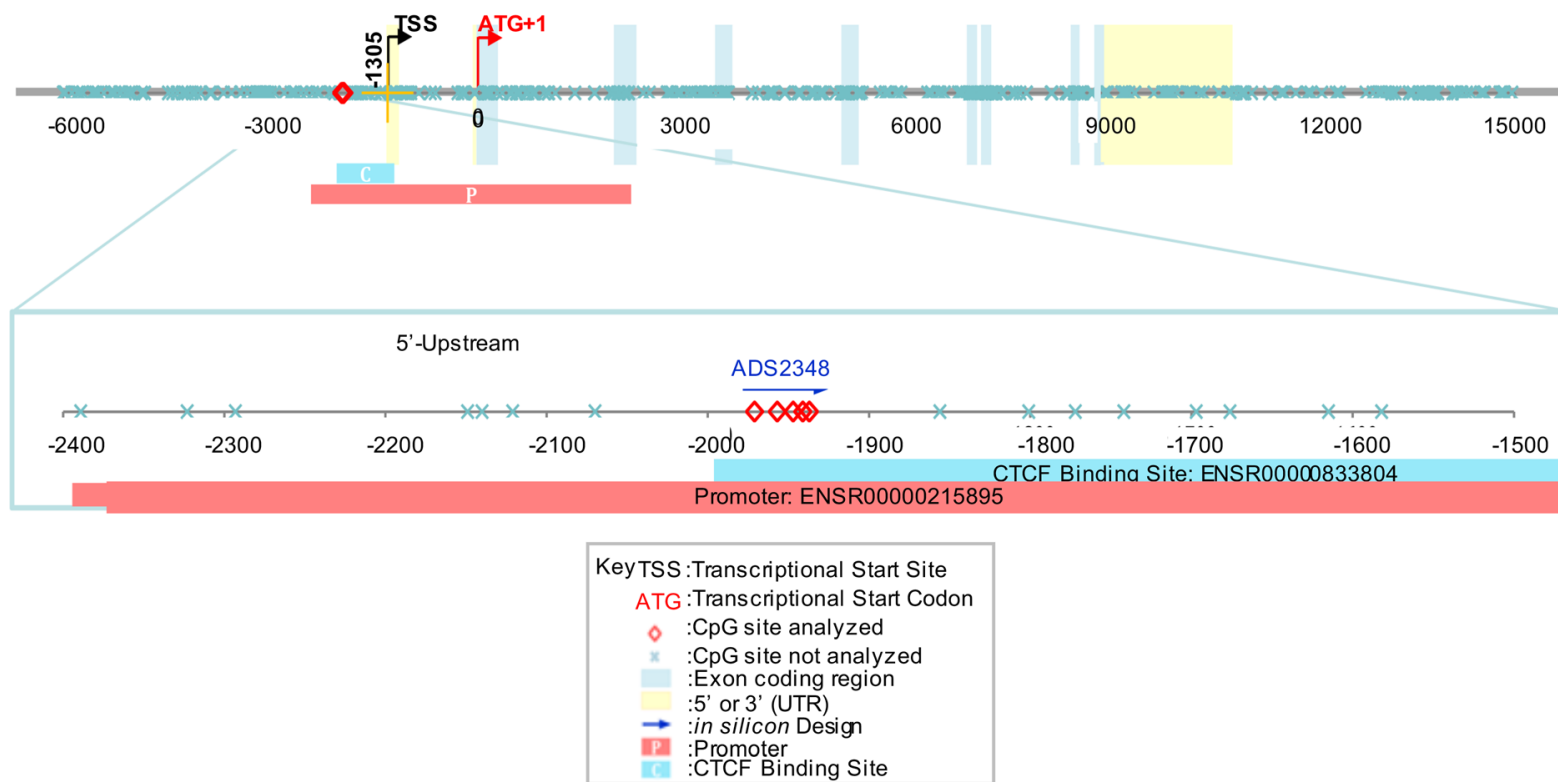

B
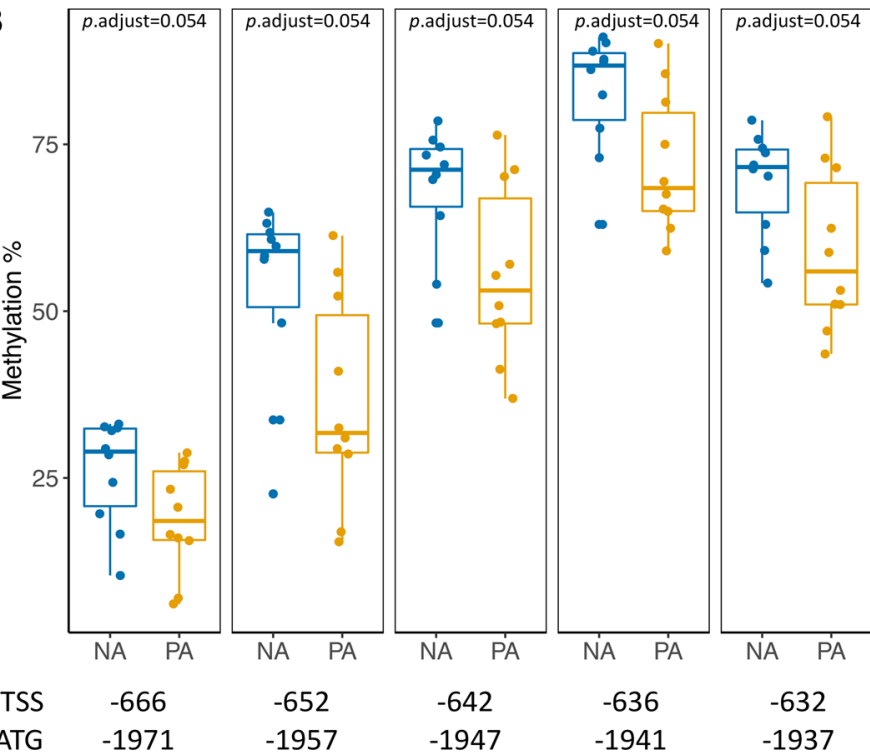

From TSS

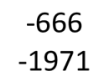

$-652$

$-1957$

$-1947$

$-1937$

Figure 2. The significant differences in the DNAm levels for $5 \mathrm{CpC}$ sites located in the region of chr7:101126423-101126457 of the SERPINE1 gene were observed between PA and NA participants. (A) Schematic of a genomic region for the SERPIN1 gene with known CpG sites. The 5 CpG sites labeled with red diamond within the targeted genomic region (chr7:101126423-101126457) were analyzed by tNGBS in our study. (B) The box plots overlaid with dot plots show the significant differences in methylation levels at each of $5 \mathrm{CpC}$ sites in targeted SERPINE1 region between PA $(n=10)$ and NA ( $n=10)$ participants (adjusted $P<0.1$ ). Each dot represents one sample. Box plots indicate the interquartile range (IQR) and median; whiskers extend to the farthest data point within a maximum of $1.5 \times$ IQR. Sample sets were analyzed using the Wilcoxon rank sum test ( 2 sided).

\section{Discussion}

This study presents potentially novel findings on the loci differentially methylated for food allergy. The PA-associated DNAm signatures at the targeted genomic regions for 12 genes were identified by comparing the DNAm levels in 125 targeted genomic regions containing $602 \mathrm{CpG}$ sites for 70 immune-related genes between PA and NA participants. These DNAm signatures for 12 genes include the genes associated with Th1/Th2 differentiation ( IL4, IL12B, and IL2), innate immunity (IL1B, ILO), and those involved with immune regulation but not specifically for food allergy, such as BDNF, IL17F, CXCL12, CCR7, RUNX1, CD3E, and 

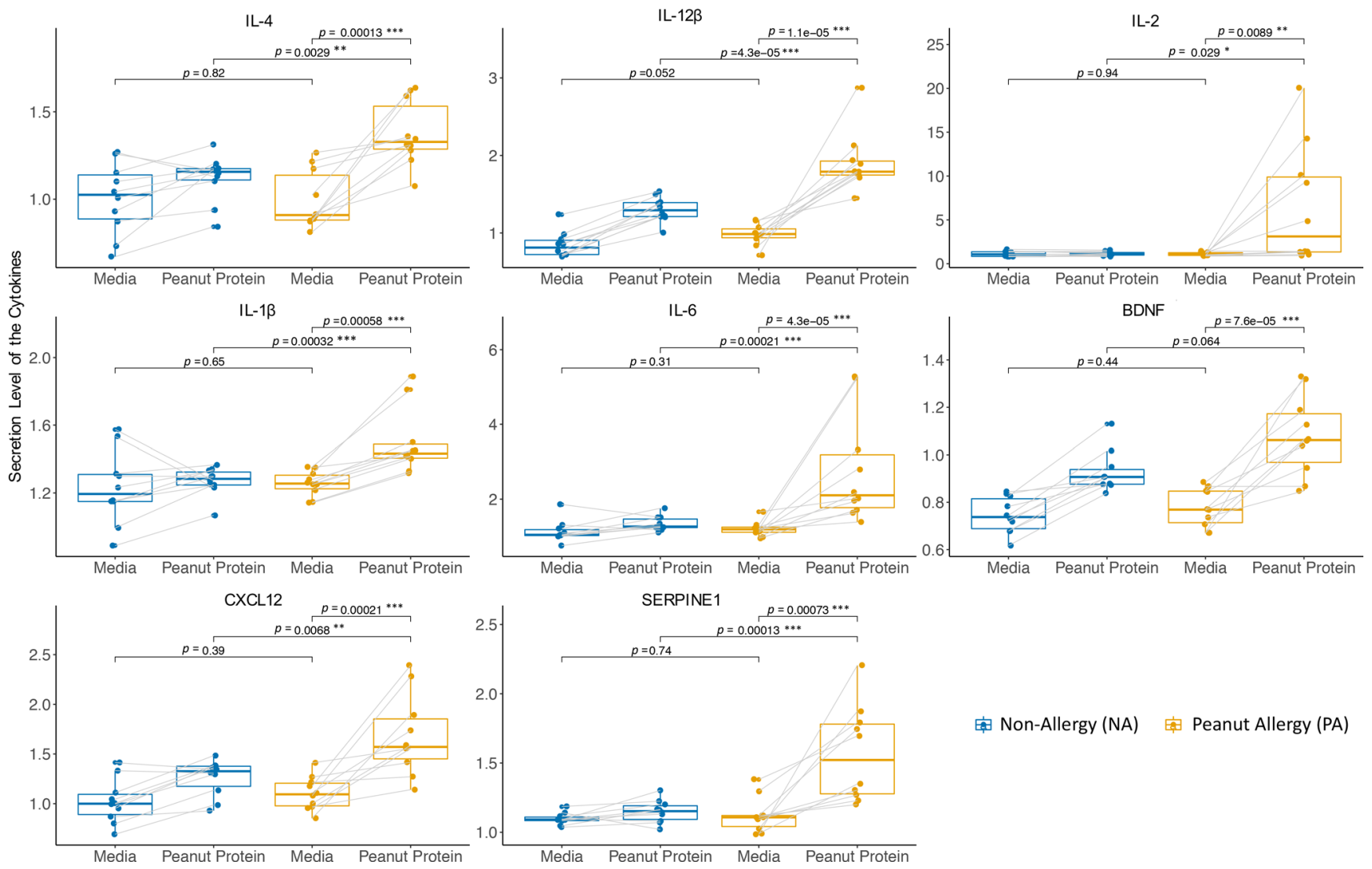

槃 Non-Allergy (NA) Peanut Allergy (PA)

Figure 3. PBMCs from PA participants secrete higher levels of cytokines (IL-4, IL-12 $\beta$, IL-2, IL-1 $\beta$, IL-6, CXCL12, and BDNF) and SERPINE1 protein compared with NA participants. Secreted levels of cytokines and SERPINE1 protein from PBMCs stimulated with or without peanut protein for PA ( $n=10)$ and NA $(n=10)$ participants are shown in box plots overlaid with dot plots $\left({ }^{*} P<0.05,{ }^{* *} P<0.01,{ }^{* * *} P<0.001\right)$. Each pair of points connected by a line represents 1 sample. Box plots indicate the IQR and median; whiskers extend to the farthest data point within a maximum of $1.5 \times$ IQR. Sets of paired samples were analyzed using the Wilcoxon signed rank test ( 2 sided). Unpaired sample sets were analyzed using the Wilcoxon rank sum test ( 2 sided).

SERPINE1. Incubating PBMCs from PA participants with peanut protein resulted in the increased secretion of IL-4, IL-12 $\beta$, IL-2, IL-1 $\beta$, IL-6, CXCL12, BDNF, and SERPINE1, compared with NA participants, which may suggest that the differences in PBMC responses on stimulation with specific food allergens between PA and NA groups are associated with DNAm changes and environmental interactions. In addition, our data show that 3 combinations of DNAm signatures from the 12 PA-associated DNAm signatures have superior diagnostic performance against serum peanut-specific IgE for discriminating PA versus NA. Our results also demonstrate that PA-associated 12 DNAm signatures are influenced by genetic factors.

It has long been understood that IgE-mediated food allergy results from a Th2 immune response of the adaptive immune system to protein antigens associated with specific foods (14). Therefore, the skewing of naive $\mathrm{CD}^{+} \mathrm{T}$ cell differentiation into $\mathrm{Th} 1$ or $\mathrm{Th} 2$ effector cells, driven by the cytokine environment, is critical to the development of food allergy. Cytokine IL-12 is mainly produced by phagocytic cells (monocytes, macrophages, neutrophils and DCs) and has been shown to drive naive T cells to differentiate into Th1 cells. IL-4 (produced by Th2 cells) is a major cytokine driving the differentiation of naive T cells into a Th2 subset (15). In addition, studies have suggested that IL-2 also has a role in facilitating Th2 differentiation (16). Low doses of IL-2-induced Treg expansion provides protection against clinical manifestation of food allergy by Treg-dependent modification of Th1/Th2 balance (17). Consistent with previous studies showing the epigenetic regulation of Th1/Th2 differentiation (18), compared with NA participants, PA participants showed a decrease in DNAm levels at the targeted genomic regions of IL4 and IL2, as well as an increase in DNAm levels at the targeted genomic region of IL12B.

The proinflammatory cytokines IL- $1 \beta$ and IL-6, mainly produced by different innate immune cells, were significantly increased in food-allergic compared with NA participants when stimulated by LPS (19) or by specific allergens, as shown in Figure 2. These observations suggest that different innate immune 
A

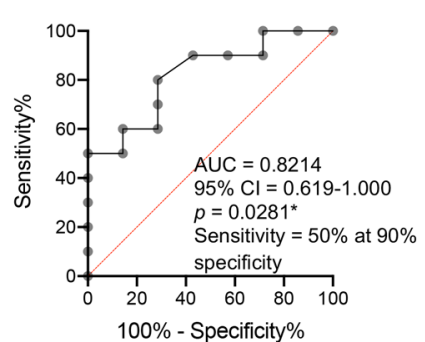

ROC curve of IL6

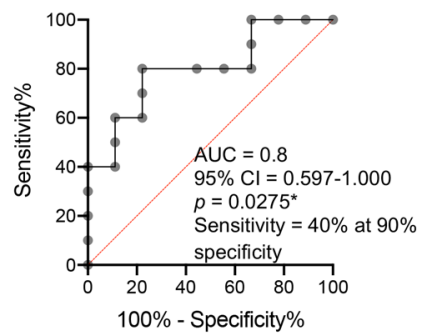

ROC curve of CCR7

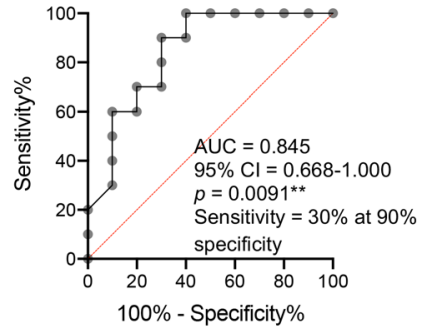

ROC curve of $I L 12 B$

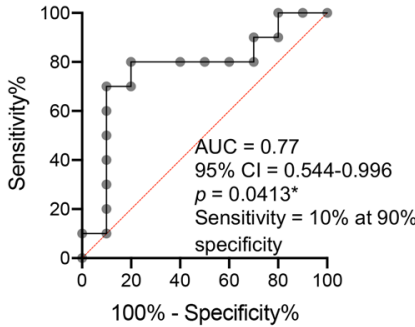

ROC curve of BDNF

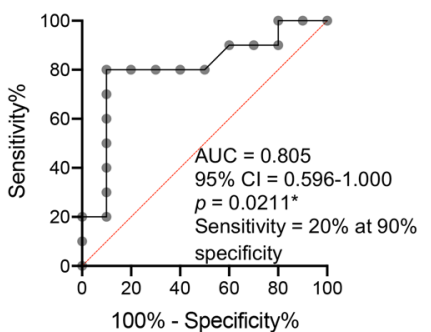

ROC curve of RUNX1

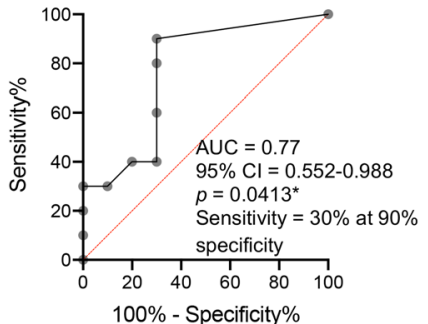

ROC curve of IL2

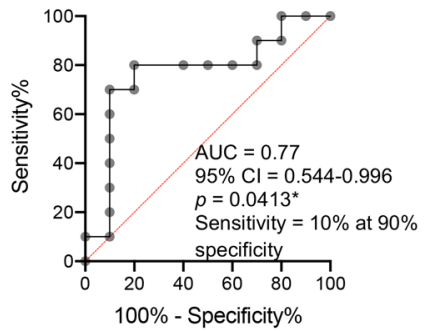

ROC curve of IL17F

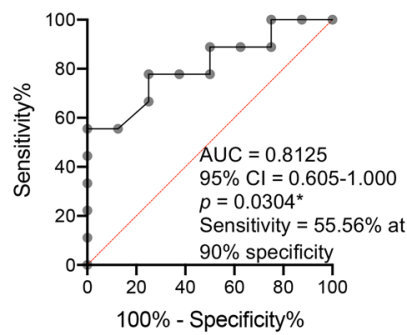

ROC curve of $C D 3 E$

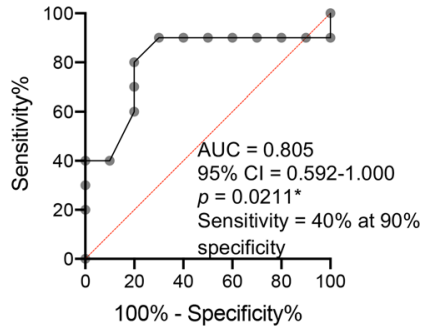

ROC curve of IL1B

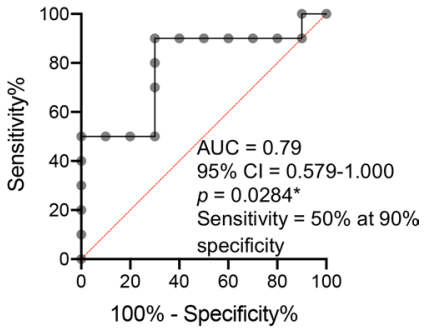

ROC curve of CXCL12

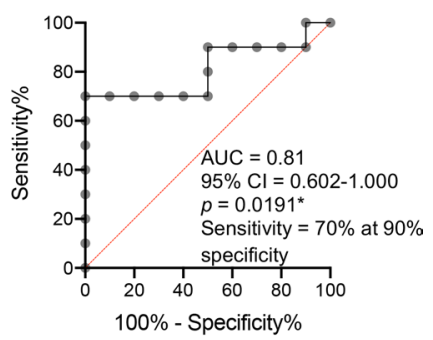

ROC curve of SERPINE1

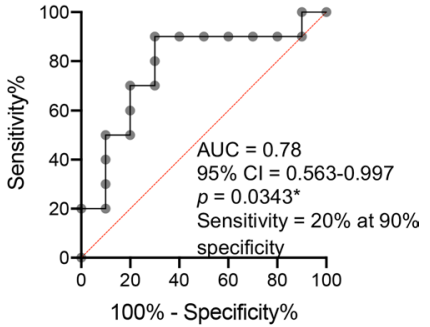

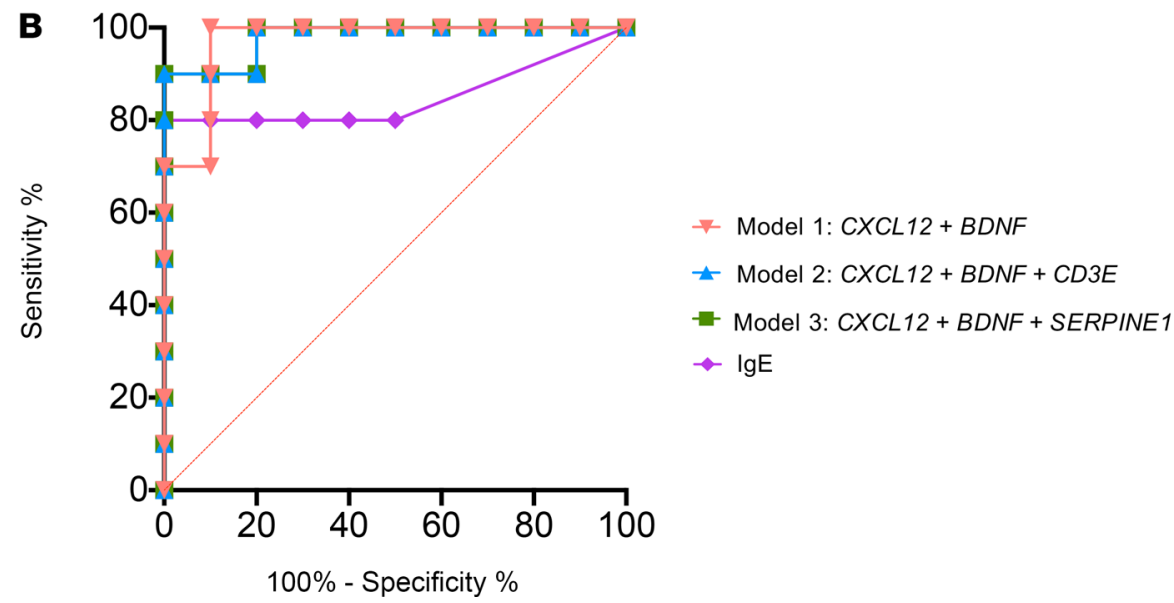

Figure 4. ROC analysis is applied to evaluate the performance of DNAm signatures for discrimination of PA versus NA groups. (A) ROC curves show the AUC and the sensitivity and specificity for each of the 12 DNAm signatures. (B) ROC curves show the AUC, and the sensitivity and specificity, for comparison of 3 models with the combination DNAm signatures against peanut-specific IgE in serum.

responses occur in food-allergic versus nonallergic participants. A study demonstrated that exposure to LPS (tolerance immunity) or bacterial $\beta$-glucan (trained immunity) induces epigenetic changes in monocytes. These reprogrammed epigenetic landscapes of innate immune cells determine the capacity for developing a "memory" in response to exogenous exposure (20). In our study, we also observed significant decreases 
Table 2. Summary of the ROC curve analysis of the 3 models with the combination DNAm signatures selected from initial 12 DNAm signatures and peanut-specific IgE in serum

\begin{tabular}{|c|c|c|c|c|c|c|}
\hline Clinical question & Predictor & AUC & $P$ value & $95 \% \mathrm{Cl}$ & $\begin{array}{c}\text { Sensitivities(\%) } \\
\text { at } 90 \% \text { specificity }\end{array}$ & AIC \\
\hline \multirow{4}{*}{$\begin{array}{c}\text { Peanut allergy } \\
\text { versus nonallergy }\end{array}$} & $\lg \mathrm{E}$ & 0.85 & 0.0082 & $0.6528 \sim 1.000$ & 80 & 14.3 \\
\hline & $C X C L 12+B D N F$ & 0.97 & 0.0004 & $0.9019 \sim 1.000$ & 70 & 13.8 \\
\hline & $C X C L 12+B D N F+C D 3 E$ & 0.98 & 0.0003 & $0.9295 \sim 1.000$ & 90 & 15.4 \\
\hline & $C X C L 12+B D N F+$ SERPINE1 & 0.98 & 0.0003 & $0.9295-1.000$ & 90 & 15.4 \\
\hline
\end{tabular}

in DNAm levels for the gene $I L 1 B$ and IL6 in PA compared with NA individuals, suggesting a link between epigenetic regulation of the innate immune system and food allergy.

It is becoming increasingly clear that immune cells do not act alone and that crosstalk and reciprocal regulation between neural and immune systems are essential in the pathophysiology of allergic diseases, including allergic asthma, atopic dermatitis, and food allergies $(21,22)$. Both immune and neural cells detect and respond to environmental threats and harmful stimuli, including allergens. Proinflammatory mediators, such as cytokines or chemokines, mediate allergic responses and also directly activate sensory neurons that regulate itch, sneezing, bronchoconstriction, and alterations in gastrointestinal motility (23). However, the mediators between neuronal and immune cells and their role in mediating allergic responses remain unclear. BDNF is a member of the neurotrophin family, which is known to be related to canonical nerve growth factor and neurogenic inflammation $(24,25)$. Recently, neurotrophins were also found to be produced continuously during allergic inflammation (26). Increased expression of BDNF has been observed in severe asthma, bronchial hyperresponsiveness, and inflammation (26), but to our knowledge, it has not been observed in food allergy. Our results, show that DNAm levels of BDNF are significantly decreased in PA compared with NA participants, suggesting that epigenetic modification of the BDNF gene is associated with food allergy. In addition, the combinations of DNAm signatures of $B D N F$ and $C X C L 12$ had the most superior diagnostic potential among all other combinations selected compared with serum peanut-specific IgE for discriminating PA versus NA.

Of note, the average DNAm level in the targeted SERPINE1 genomic region (chr7:101126423101126457) and the DNAm levels at each of $5 \mathrm{CpG}$ sites in this targeted region were significantly decreased in PA compared with NA participants. In mice, following intranasal OVA challenge, WT

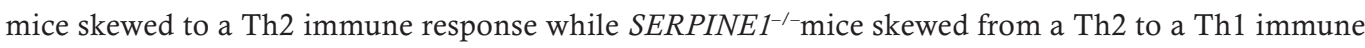
response (27). In house dust mite (HDM) allergic asthma patients, SNP (rs1799768) in the SERPINE1 gene was associated with bronchial reactivity to histamine and IgE response (28). Interestingly, the gene SERPINE1 5'-upstream promoter region (chr7:101126423-101126457; Figure 1C) contains the allergic disease-associated SNP (rs1799768, chr7:101126426) (28). It has been proposed that epigenetic modification may mediate the effect of genetic variants on the development of food allergy (3). For example, the DNAm of HLA-DQB1 and HLA-DRB1 genes were implicated in the mediation of the association between SNPs in HLA-DQ and PA and between HLA-DR and PA (29). Consistent with this possibility, the observation on the genomic location of allergy-associated SERPINE1 SNP suggests that the DNAm in the above targeted regions for the SERPINE1 gene might act as a mediator of the association between genetic variation and allergic disease.

IL-17F and IL-17A are related homodimeric proteins of the IL-17 family and are produced by Th17 cells. Both $17 \mathrm{~F}$ and IL-17A cytokines have been implicated in allergic inflammation (21) and inflammation resulting from mucosal immunity or autoimmunity (30). It has been suggested that IL-17A plays a role in the regulation of food allergy and is a potential biomarker of tolerance to food allergens (31); however, the role of IL-17F in food allergy is not well understood. Our results suggest that epigenetic modification of the $I L 17 F$ gene is associated with food allergy.

Increased levels of the chemokine CXCL12 has been found in the bronchoalveolar lavage of patients with asthma (32). Overexpression of the chemokine receptor CCR7 in DC is thought to mediate DC lymph node migration and promote the development of allergic responses (33). Genome-wide association studies (GWAS) show that the SNPs in RUNX1 are associated with airway responsiveness in asthmatic 
A

\begin{tabular}{l|c|c|c|} 
& MZPA & All PA & All NA \\
\hline MZ NA & $15.88 *$ & 23.86 & 27.92 \\
\hline All NA & 28.64 & $30.11 \mathrm{~s}$ & \\
All PA & 17.72 & &
\end{tabular}

B

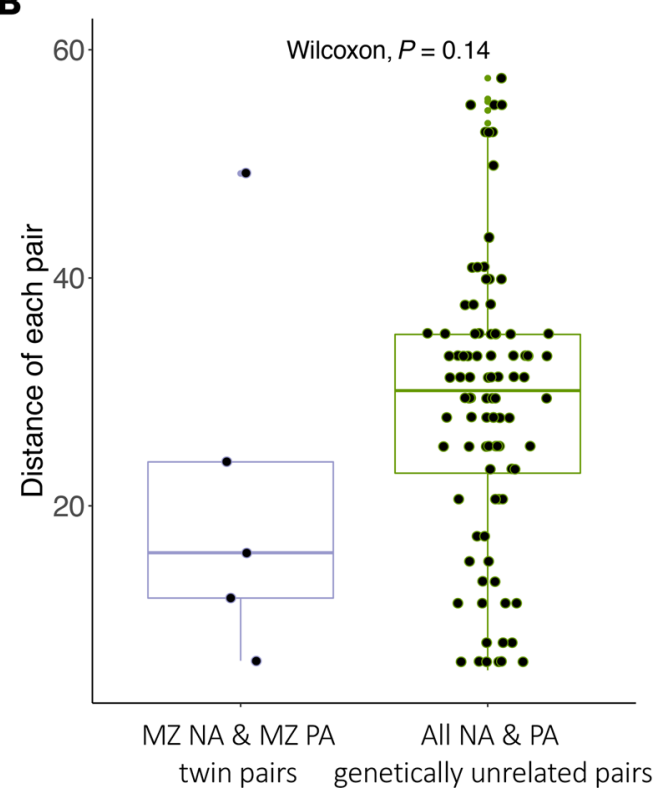

C

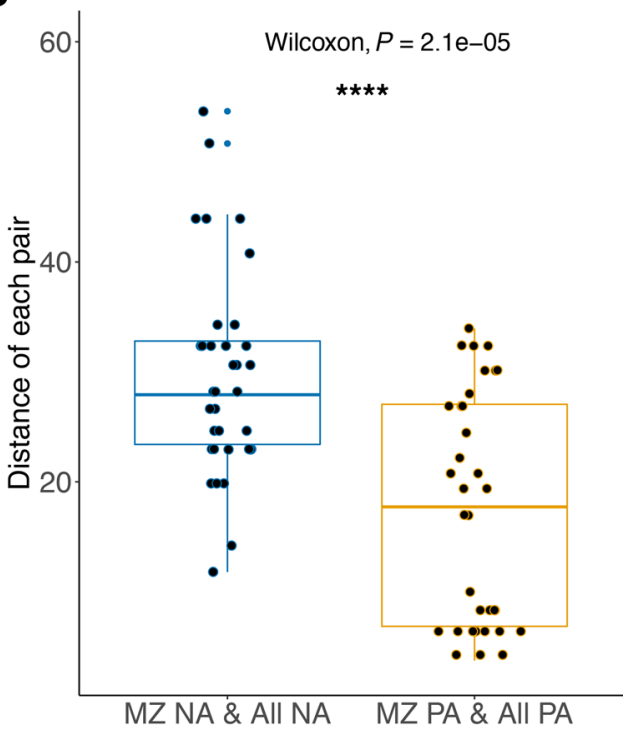

Figure 5. Peanut allergy-associated DNAm levels in 12 targeted genomic regions are genetically influenced. (A) Euclidean distances of 12 DNAm signatures were calculated pairwise either between 5 MZ twin pairs (5 pairs) who are discordant for peanut allergy or randomly selected genetically unrelated pairs (95 pairs) (i.e., 1 sample has peanut allergy and the other is nonallergy without peanut allergy). "Median of the distance within 5 discordant MZ twin pairs. ${ }^{5}$ Median of the distance of all 95 genetically unrelated NA and PA pairs. (B) Box plots overlaid with dot plots represent the Euclidean distances between MZ PA and NA twin pairs (pairs = 5 , left panel) and genetically unrelated individuals in PA participants and in NA individuals (pairs = 95, right panel). (C) Box plots overlaid with dot plots represent the Euclidean distances between random pairs, of which 1 of each pair is an NA MZ twin and the other is a genetically unrelated NA individual (pairs = 35, left panel), and random pairs, of which 1 of each pair is a PA MZ twin and the other is a genetically unrelated PA individual (pairs = 35, right panel). Box plots indicate the interquartile range (IQR) and median; whiskers extend to the farthest data point within a maximum of $1.5 \times$ IQQR. The Wilcoxon rank sum test $(2$ sided) was used for comparison analysis. ${ }^{* * *} P<0.001$.

children (34), and the RUNX1 transcription factor has been identified as a molecular link in TGF- $\beta$ induced FOXP3 expression in inducible Treg (35). It has been proposed that the signals transduced by $\mathrm{CD} 3 \varepsilon$ contribute quantitatively to TCR signaling and that $\mathrm{CD} 3 \varepsilon$ signals suggest a potential role in the generation and/or survival of mature T cells (36). Here, our results show the association between DNAm levels with food allergy for CXCL12, CCR7, RUNX1, and CD3E.

DNAm is most commonly associated with downregulation of gene expression - especially when the hypermethylation is in the promotor region of a gene. In our study, IL4,IL2, IL1B, IL6, BDNF, and SERPINE1 showed lower methylation level and higher protein level in the PA compared with NA participants. However, the PA patients have increased DNAm at 5' upstream of $I L 12 B$ and CXCL12, which is associated with increased IL-12 $\beta$ and CXCL12 protein expression compared with NA participants. It should be noted that, although gene silencing by promoter hypermethylation seems to be the most likely mode of action, there is growing evidence of a more complex view on the effect of DNAm in various contexts (37-41). In particular, for genes that become methylated, the associated expression level can be unaffected or even upregulated in some cases (40), suggesting a more diverse mechanism of epigenetic regulation. Such additional complexity could have important implications for understanding allergic disease but has not been studied at a genome-wide scale. 
Among the $10 \mathrm{PA}$ and 10 NA participants in this study, 7 are allergic to tree nuts, 4 have asthma, and 6 have atopic dermatitis. To examine whether similar DNAm signatures are presented in other allergic disease, we applied the Wilcoxon rank sum test to compare the average $\mathrm{CpG}$ methylation levels within individual targeted genomic regions using our original data (total 125 targeted genomic regions covering $602 \mathrm{CpG}$ sites) between 6 atopic dermatitis versus 14 nondermatitis participants, 4 asthma versus 16 nonasthma participants, and 7 tree nut-allergic versus 13 non-tree nut-allergic participants. The differential methylated genomic regions related to atopic dermatitis, asthma, or tree nut allergy are presented in Supplemental Figures 2-4. Among the 10 differentially methylated genomic regions associated with atopic dermatitis (Supplemental Figure 2), none of them overlap with the $12 \mathrm{PA}$-associated differentially methylated genomic regions, and all of the 10 genes have been reported to be associated with atopic dermatitis in previous studies (42-51). Of note, the differential DNAm in the genomic region of the filaggrin gene $(F L G)$, which plays an important role in the pathogenesis of atopic dermatitis and allergic disease $(42,52)$, is identified in our comparison analysis between nonatopic dermatitis and atopic dermatitis participants. Among 4 differentially methylated genomic regions associated with asthma (Supplemental Figure 3), 2 of them $(I L 12 B, I L 2)$ overlap with the 12 PA-associated differentially methylated genomic regions, and all of the 4 genes have been reported to be associated with asthma in previous studies (53-57). Among 9 differentially methylated genomic regions associated with tree nut allergy (Supplemental Figure 4), only 1 (CXCL12) overlaps with the 12 PA-associated differential methylated genomic regions, and 6 of the 9 genes (IL10, IKZF2, CCL5, IL21, CCR9, and IL-33) have been reported to be associated with food allergy in previous studies (58-63). All of the above results support that the 12 differentially methylated genomic regions are, to some extent, specific to PA.

Oral food challenges (OFCs) are the gold standard for diagnosis of food allergy; however, OFCs are associated with risk of allergic reaction and, therefore, need to be performed in clinics with trained staff; this practice limits widespread use (64). The study by Martino and colleagues (8) showed that DNAm signatures at $96 \mathrm{CpG}$ sites can predict food challenge outcomes by comparing the differences of DNAm between food allergic and food sensitization groups. Their results show that the 73 genes overlapped by these $96 \mathrm{CpG}$ sites were enriched in the sole MAP kinase canonical pathway but were not involved in the well-known food allergy-associated Th1/Th2 pathway. In addition, these clinically relevant biomarkers have not been widely used in clinic diagnosis. We also compared the diagnostic performance for these 12 DNAm signatures for PA against peanut-specific IgE in serum. We found that diagnostic sensitivity for peanut-specific IgE at $90 \%$ specificity is $80 \%$, but the sensitivity of individual DNAm signatures at $90 \%$ specificity ranged from $10 \%$ to $70 \%$. To select the optimal combination of DNAm signatures from the initial 12 DNAm signatures, we further performed stepwise regression analysis and ROC curve analysis, and our pilot exploratory results indicate that the combinations of DNAm signatures selected from the initial 12 DNAm signatures had superior diagnostic potential compared with serum peanut-specific IgE for discriminating PA versus NA.

The birth rate for $\mathrm{MZ}$ twins is about $0.3 \%$ of the world population, and a previous study showed that, among 14 pairs of MZ twins, only 5 of them were discordant for PA (65). The young allergy-discordant MZ twin participants used in this study have nearly perfect controls of covariates such as age, sex, and genetic and environmental factors, which increase the rigor and reproducibility of our epigenetic association studies. It increases the power estimation over ordinary case-control designs by minimizing confounding genetic and environmental factors $(66,67)$. In addition, to avoid reporting findings caused by natural variability within twin pairs, we compared our data with a study that investigated genome-wide DNAm variability in adolescent $\mathrm{MZ}$ twins followed since birth (68). This study showed that probes with the highest within-pair differences in DNAm were enriched in gene ontologies related to development and cell growth. The PA-associated 12 differential methylated gene regions identified in our study do not overlap with the hypervariable genes across MZ twins in previous studies (68). The lack of overlap between the 2 studies, in gene variability within twin pair differences in DNAm, reduces the likelihood that our findings were due to general within-pair variability and increases our confidence that these data represent true methylation difference associated with PA. The development of these combinations of DNAm signatures for diagnosis of PA need further verification in detailed follow-up studies with large sample size.

Overall, our results reveal that the food allergy-associated DNAm signatures suggest epigenetic modifications could discern PA versus NA individuals, and that these DNAm signatures could be potentially used for diagnostics or future medical research in not only PA, but also food allergy. Several DNAm signatures highlighted in the current study have been associated with PA, suggesting that these genes may have 
important roles in food allergy with potential as biomarkers and potential for targeted therapy. Further validation with a larger cohort and further functional studies are warranted to enable further understanding of the molecular mechanisms underpinning food allergy. The potentially novel PA-associated DNAm genes, such as BDNF (neurotrophin) and SERPINE1 (serine protease inhibitor), suggest an additional direction of research for deciphering the molecular basis of PA. Furthermore, a high similarity between PA and NA participants was observed in MZ twins compared with randomly paired genetically unrelated individuals, indicating that the PA-associated 12 DNAm signatures were genetically influenced.

\section{Methods}

Study participants. PA discordant MZ twin siblings, nontwin PA pediatric participants, and nontwin NA pediatric participants were recruited at the Sean N. Parker Center for Allergy and Asthma Research at Stanford University. Patient demographics, food allergy history, atopic history, and peanut-specific IgE are summarized in Table 1. PA was confirmed by a food challenge by a certified allergy specialist. Blood specimens were drawn before food challenges were performed, and no participant was taking any medications (e.g., steroids, valproic acid, folic acid, DNA intercalating agents, methotrexate, or DNA methyltransferase inhibitors) that could have affected the epigenetics.

Collection and processing of blood specimens. PBMCs and plasma were isolated from blood samples by density gradient centrifugation (400g for 20 minutes at room temperature) over Ficoll-Paque. PBMCs were cryopreserved in $10 \%$ dimethyl sulfoxide in FCS and stored in liquid nitrogen. Plasma was stored at $-80^{\circ} \mathrm{C}$.

$t N G B S$. tNGBS was performed on PBMCs from 10 PA and 10 NA participants by EpigenDx Inc. In total, 125 targeted genomic regions containing $602 \mathrm{CpG}$ sites for 70 genes were analyzed using tNGBS. Targeted bisulfite deep-sequencing PCR products were purified using QIAquick PCR purification kit (QIAGEN). Ion Torrent deep-sequencing libraries were constructed from bisulfite-converted DNA using the KAPA Library Preparation Kit (Kapa Biosystems), quantified using the QIAxcel Advanced System (QIAGEN), templated using the Ion PGM Template OT2 200 kit (Thermo Fisher Scientific), and sequenced using the Ion PGM Sequencing Hi-Q OT2 Kit with the Ion 314 Chip Kit v2 on an Ion PGM System (Thermo Fisher Scientific), which generated nondirectional, approximately $200 \mathrm{nt}-$ length reads at 1500-7500 reads per library in the fastq format. FASTQ files from the Ion PGM System were filtered and aligned to the human genome assembly hg38 using Bismark Bisulfite Mapper v0.15.0 (Babraham Bioinformatics) with the Bowtie 2 alignment algorithm. Methylation levels were calculated in Bismark by dividing the number of cytosine converted (cytosine versus thymine; $\mathrm{C}$ versus $\mathrm{T}$ ) reads by the number of total reads, considering all $\mathrm{CpG}$ sites covered by a minimum of 30 total reads. The DNAm level at each of $602 \mathrm{CpG}$ sites were reported by EpigenDx.

In vitro stimulation. The peanut proteins added to cell culture were derived from peanut flour used for double-blind placebo-controlled food challenges (DBPCFCs) in the clinic. The peanut flour was dissolved in PBS and sterilized by filtration. The peanut protein concentration was determined by BCA Protein Assay (Pierce). The endotoxin level of peanut protein was assessed by fluorescence-based rFC assay (Indoor Biotechnologies), and the endotoxin level of peanut protein exhibited in cell culture was $0.05 \mathrm{EU} / \mathrm{mL}$.

After overnight resting of thawed PBMCs, cells were cultured in complete RPMI medium (RPMI1640 medium [Thermo Fisher Scientific], glutamine [Thermo Fisher Scientific], 5\% human serum [MilliporeSigma], 1\% penicillin/streptomycin [Thermo Fisher Scientific]) and in the presence or absence of peanut protein at a final concentration of $100 \mu \mathrm{g} / \mathrm{mL}$ for 3 days. For each condition of this experiment, PBMCs were cultured at $5 \times 10^{5}$ cells per $200 \mu \mathrm{L}$ for 3 days, after which the supernatants were harvested and stored at $-80^{\circ} \mathrm{C}$.

Cytokine assays. The secretion levels of cytokines or chemokines from PBMCs in supernatants were measured using a 62-multiplex assay on the Luminex 200 IS system (Affymetrix) performed by Stanford Human Immune Monitoring Center (HIMC). All samples were tested in duplicate wells. Data were analyzed using MasterPlex software (Hitachi Software Engineering America Ltd., MiraiBio Group), and the average of 2 median fluorescence intensity (MFI) values for each sample for each analyte were reported by Stanford HIMC. Then, the ratios were calculated by dividing the average MFI of each analyte for each sample by the average MFI of each analyte for complete RPMI medium control. These ratios were used to present the secretion level of each cytokine or chemokine from PBMCs for each sample.

Flow cytometry. PBMCs from 10 NA and 10 PA participants were stained with the lineage markers listed in Supplemental Table 5 to examine the proportion of major immune cell subtypes in total PBMCs. The PBMCs (about $1 \times 10^{6}$ cells per sample) were incubated in $100 \mu \mathrm{L}$ volume with Human TruStain FcX (BioLegend), cell surface antibodies (in Supplemental Table 5) and viability dyes (Aqua, 
Thermo Fisher Scientific) for 30 minutes on ice, and followed by washing 2 times with FACS buffer (PBS with $0.25 \%$ BSA and $1 \mathrm{mM}$ EDTA). All samples were run on LSRII flow cytometer and analyzed using FlowJo Version 10.6.0 software.

Statistics. The analysis was conducted using the statistical programming language R (version R 3.6.2). The differences in average methylation across all $\mathrm{CpG}$ in each targeted genomic region and the differences in DNAm level at each CpG site in individual targeted genomic region, were compared between PA and NA participants using Wilcoxon rank sum test (nonparametric unpaired test, 2 sided). A $P$ value less than 0.05 was considered significant. Using the Benjamini-Hochberg procedure with an FDR of 0.1, the assay-wise multiple testing correction was further performed across each of the $\mathrm{CpG}$ sites in individual targeted genomic region (or individual independent assay). An adjusted $P$ value less than 0.1 was considered significant

The mixed-effects logistic regression correcting for the dependent participants, such as MZ twin samples, was conducted using the "glmer" function in the "lme4" R package (version 1.1-23). The DNAm signatures used for regression and creating the ROC curve are the average methylation levels across the CpG sites within a targeted genomic region, which were detected by tNGBS by 1 pair of primers. The step-up regression was performed to select the combination of variables in terms of the AIC value and AUC value. Three models with the combinations of 2 or 3 methylation signatures have the both top 3 lowest AIC values and top 3 highest AUC values. Equivalent ROC curves were obtained from mixed-effects logistic regression. ROC analysis was performed using Prism 8 (GraphPad).

PCA was then conducted to visualize the DNAm signatures between NA and PA groups using pcaMethods package in R. PERMANOVA was applied to examine the contribution of variables to the separation of the data in multiple dimensional space using the "adonis2" function in the "vegan" package in R. The distance function in R software was employed for Euclidean distances analysis. All dot plots overlaid with boxplots or line connections were compiled with ggplot2 package in $\mathrm{R}$.

Study approval. The study was approved by the IRB of Stanford University and registered at Clinicaltrials.gov (NCT01613885). All participants or their caregivers provided written informed consent.

\section{Author contributions}

KCN conceived the study. XZ and SCL performed experiments and collected data. BB and LK contributed to the recruitment of study participants. IC contributed to processed samples for this study. XZ, XH, SCL, and $\mathrm{KCN}$ contributed to experimental design, data analysis, and data interpretation. $\mathrm{SC}$ assisted with statistical analyses. XZ, XH, VS, and KCN wrote the paper. XZ and XH contributed equally to this research as co-first authors. The order of appearance of the co-first authors was based on the timeline of contributions to the work.

\section{Acknowledgments}

We acknowledge Holden T. Maecker for his advice on the analysis of Luminex data, as well as the Stanford Twin Registry. This work was supported by grants from NIAID (R01AI140134), NHLBI (R01HL118612), the Sean N. Parker Center for Allergy and Asthma Research at Stanford University, and The Stanford Institute for Immunity, Transplantation and Infection (ITI) Seed Grant.

Address correspondence to: Kari C. Nadeau, Sean N. Parker Center for Allergy and Asthma Research at Stanford University, 240 Pasteur Dr. BMI Rm.1755, Palo Alto, California 94304, USA. Phone: 650.867.4592; Email: knadeau@stanford.edu.

1. Gupta RS, et al. Prevalence and severity of food allergies among US adults. JAMA Netw Open. 2019;2(1):e185630.

2. Gupta RS, et al. The public health impact of parent-reported childhood food allergies in the United States. Pediatrics. 2018;142(6):e20181235.

3. Bar-El Dadon S, Reifen R. Chapter 55 - Epigenetics in Food Allergies: The Missing Piece of the Puzzle. In: Caterina RDE, Martinex AJ, Kohlmeier M, eds. Principles of Nutrigenetics and Nutrigenomics. Elsevier; 2020:403-409.

4. Hong X, Wang X. Epigenetics and development of food allergy (FA) in early childhood. Curr Allergy Asthma Rep. 2014;14(9):460.

5. Kabesch M, Tost J. Recent findings in the genetics and epigenetics of asthma and allergy. Semin Immunopathol. 2020;42(1):43-60.

6. Berni Canani R, et al. Differences in DNA methylation profile of Th1 and Th2 cytokine genes are associated with tolerance acquisition in children with IgE-mediated cow's milk allergy. Clin Epigenetics. 2015;7:38.

7. Syed A, et al. Peanut oral immunotherapy results in increased antigen-induced regulatory T-cell function and hypomethylation of forkhead box protein 3 (FOXP3). J Allergy Clin Immunol. 2014;133(2):500-510. 
8. Martino D, et al. Blood DNA methylation biomarkers predict clinical reactivity in food-sensitized infants. J Allergy Clin Immunol. 2015;135(5):1319-1328.

9. Martino D, et al. Epigenetic dysregulation of naive CD4+ T-cell activation genes in childhood food allergy. Nat Commun. 2018;9(1):3308

10. Prunicki M, et al. Exposure to $\mathrm{NO}_{2}, \mathrm{CO}$, and $\mathrm{PM}_{25}$ is linked to regional DNA methylation differences in asthma. Clin Epigenetics. 2018;10:2

11. Fraga MF, et al. Epigenetic differences arise during the lifetime of monozygotic twins. Proc Natl Acad Sci US A. 2005;102(30):10604-10609.

12. Cheung $\mathrm{P}$, et al. Single-cell chromatin modification profiling reveals increased epigenetic variations with aging. Cell. 2018;173(6):1385-1397.

13. Ollikainen M, et al. DNA methylation analysis of multiple tissues from newborn twins reveals both genetic and intrauterine components to variation in the human neonatal epigenome. Hum Mol Genet. 2010;19(21):4176-4188.

14. Yu W, et al. Food allergy: immune mechanisms, diagnosis and immunotherapy. Nat Rev Immunol. 2016;16(12):751-765

15. Dong C, Flavell RA. Cell fate decision: T-helper 1 and 2 subsets in immune responses. Arthritis Res. 2000;2(3):179-188.

16. Le Gros G, et al. Generation of interleukin 4 (IL-4)-producing cells in vivo and in vitro: IL-2 and IL-4 are required for in vitro generation of IL-4-producing cells. J Immunol. 2008;181(5):2943-2951.

17. Bonnet B, et al. Low-dose IL-2 induces regulatory T cell-mediated control of experimental food allergy. J Immunol. 2016;197(1):188-198.

18. Hong X, Wang X. Early life precursors, epigenetics, and the development of food allergy. Semin Immunopathol. 2012;34(5):655-669. 19. Neeland MR, et al. Early life innate immune signatures of persistent food allergy. J Allergy Clin Immunol. 2018;142(3):857-864.

20. Novakovic B, et al. $\beta$-glucan reverses the epigenetic state of LPS-induced immunological tolerance. Cell. 2016;167(5):1354-1368

21. Wang YH, Wills-Karp M. The potential role of interleukin-17 in severe asthma. Curr Allergy Asthma Rep. 2011;11(5):388-394.

22. Veres TZ, et al. The role of neuro-immune cross-talk in the regulation of inflammation and remodelling in asthma. Pharmacol Ther. 2009;122(2):203-214.

23. Nassenstein C, et al. New aspects of neuroinflammation and neuroimmune crosstalk in the airways. J Allergy Clin Immunol. 2018;142(5):1415-1422.

24. Lindsay RM, Harmar AJ. Nerve growth factor regulates expression of neuropeptide genes in adult sensory neurons. Nature. 1989;337(6205):362-364.

25. Nadel JA. Neutral endopeptidase modulates neurogenic inflammation. Eur Respir J. 1991;4(6):745-754.

26. Nockher WA, Renz H. Neurotrophins and asthma: novel insight into neuroimmune interaction. J Allergy Clin Immunol. 2006;117(1):67-71.

27. Sejima T, et al. Protection of plasminogen activator inhibitor-1-deficient mice from nasal allergy. J Immunol. 2005;174(12):8135-8143.

28. Pampuch A, et al. The - 675 4G/5G plasminogen activator inhibitor-1 promoter polymorphism in house dust mite-sensitive allergic asthma patients. Allergy. 2006;61(2):234-238.

29. Hong X, et al. Genome-wide association study identifies peanut allergy-specific loci and evidence of epigenetic mediation in US children. Nat Commun. 2015;6:6304.

30. Dubin PJ, Kolls JK. Interleukin-17A and interleukin-17F: a tale of two cytokines. Immunity. 2009;30(1):9-11.

31. Dhuban KB, et al. Altered T helper 17 responses in children with food allergy. Int Arch Allergy Immunol. 2013;162(4):318-322.

32. Chen $\mathrm{H}$, et al. CXCR4 inhibitor attenuates allergen-induced lung inflammation by down-regulating MMP-9 and ERK1/2. Int $J$ Clin Exp Pathol. 2015;8(6):6700-6707.

33. Gill MA. The role of dendritic cells in asthma. J Allergy Clin Immunol. 2012;129(4):889-901.

34. Haley KJ, et al. RUNX transcription factors: association with pediatric asthma and modulated by maternal smoking. $A m J$ Physiol Lung Cell Mol Physiol. 2011;301(5):L693-L701.

35. Klunker S, et al. Transcription factors RUNX1 and RUNX3 in the induction and suppressive function of Foxp3+ inducible regulatory T cells. J Exp Med. 2009;206(12):2701-2715.

36. Sommers CL, et al. Function of CD3 epsilon-mediated signals in T cell development. J Exp Med. 2000;192(6):913-919.

37. Marzese DM, Hoon DS. Emerging technologies for studying DNA methylation for the molecular diagnosis of cancer. Expert Rev Mol Diagn. 2015;15(5):647-664.

38. Medvedeva YA, et al. Effects of cytosine methylation on transcription factor binding sites. BMC Genomics. $2014 ; 15: 119$.

39. Yang M, Park JY. DNA methylation in promoter region as biomarkers in prostate cancer. Methods Mol Biol. 2012;863:67-109.

40. Wan J, et al. Characterization of tissue-specific differential DNA methylation suggests distinct modes of positive and negative gene expression regulation. BMC Genomics. 2015;16:49.

41. Rauluseviciute I, et al. DNA hypermethylation associated with upregulated gene expression in prostate cancer demonstrates the diversity of epigenetic regulation. BMC Med Genomics. 2020;13(1):6.

42. O'Regan GM, et al. Filaggrin in atopic dermatitis. J Allergy Clin Immunol. 2008;122(4):689-693.

43. Navarini AA, et al. Interrupting IL-6-receptor signaling improves atopic dermatitis but associates with bacterial superinfection. J Allergy Clin Immunol. 2011;128(5):1128-1130.

44. Suarez-Farinas M, et al. RNA sequencing atopic dermatitis transcriptome profiling provides insights into novel disease mechanisms with potential therapeutic implications. J Allergy Clin Immunol. 2015;135(5):1218-1227.

45. Roesner LM, et al. Foxp3(+) regulatory T cells are expanded in severe atopic dermatitis patients. Allergy. 2015;70(12):1656-1660.

46. Kaburagi Y, et al. Enhanced production of CC-chemokines (RANTES, MCP-1, MIP-1alpha, MIP-1beta, and eotaxin) in patients with atopic dermatitis. Arch Dermatol Res. 2001;293(7):350-355.

47. Yamamoto N, et al. Heterogeneity of interleukin 5 genetic background in atopic dermatitis patients: significant difference between those with blood eosinophilia and normal eosinophil levels. J Dermatol Sci. 2003;33(2):121-126.

48. Namkung JH, et al. An association between IL-9 and IL-9 receptor gene polymorphisms and atopic dermatitis in a Korean population. J Dermatol Sci. 2011;62(1):16-21.

49. Paradis TJ, et al. Essential role of CCR6 in directing activated T cells to the skin during contact hypersensitivity. J Invest Dermatol. 2008;128(3):628-633. 
50. Homey B, et al. Cytokines and chemokines orchestrate atopic skin inflammation. J Allergy Clin Immunol. 2006;118(1):178-189.

51. Barnes KC. An update on the genetics of atopic dermatitis: scratching the surface in 2009. J Allergy Clin Immunol. 2010;125(1):16-29.

52. O'Regan GM, Irvine AD. The role of filaggrin loss-of-function mutations in atopic dermatitis. Curr Opin Allergy Clin Immunol. 2008;8(5):406-410.

53. Boonpiyathad S, et al. Interleukin-2 levels in exhaled breath condensates, asthma severity, and asthma control in nonallergic asthma. Allergy Asthma Proc. 2013;34(5):e35-e41.

54. Murakami Y, et al. TLR9-IL-2 axis exacerbates allergic asthma by preventing IL-17A hyperproduction. Sci Rep. 2020;10(1):18110

55. Randolph AG, et al. The IL12B gene is associated with asthma. Am J Hum Genet. 2004;75(4):709-715.

56. Manni ML, Alcorn JF. The enigmatic role of IL-22 in asthma. Expert Rev Respir Med. 2016;10(6):619-623

57. Hwang S, et al. A protein interaction network associated with asthma. J Theor Biol. 2008;252(4):722-731.

58. Kim E, et al. Intestinal epithelial cells regulate gut eotaxin responses and severity of allergy. Front Immunol. 2018;9:1692.

59. Kishida T, et al. IL-21 induces inhibitor of differentiation 2 and leads to complete abrogation of anaphylaxis in mice. J Immunol. 2007;179(12):8554-8561.

60. Pathak M, et al. CCR9 signaling in dendritic cells drives the differentiation of Foxp $3^{+}$Tregs and suppresses the allergic IgE response in the gut. Eur J Immunol. 2020;50(3):404-417.

61. Galand C, et al. IL-33 promotes food anaphylaxis in epicutaneously sensitized mice by targeting mast cells. J Allergy Clin Immunol. 2016;138(5):1356-1366.

62. HayGlass KT, et al. Distinct roles of IL-10 in regulation of peanut allergy in humans. J Allergy Clin Immun. 2009;123(2):S27.

63. Sugita K, et al. Generation of Helios reporter mice and an evaluation of the suppressive capacity of Helios $(+)$ regulatory $\mathrm{T}$ cells in vitro. Exp Dermatol. 2015;24(7):554-556

64. Santos AF, Lack G. Food allergy and anaphylaxis in pediatrics: update 2010-2012. Pediatr Allergy Immunol. 2012;23(8):698-706.

65. Sicherer SH, et al. Genetics of peanut allergy: a twin study. J Allergy Clin Immunol. 2000;106(1 Pt 1):53-56.

66. $\mathrm{Li} \mathrm{W}$, et al. On the power of epigenome-wide association studies using a disease-discordant twin design. Bioinformatics. 2018;34(23):4073-4078

67. Sahu M, Prasuna JG. Twin studies: a unique epidemiological tool. Indian J Community Med. 2016;41(3):177-182.

68. Levesque ML, et al. Genome-wide DNA methylation variability in adolescent monozygotic twins followed since birth. Epigenetics. 2014;9(10):1410-1421 